\title{
The Effect of Social Health Issues on Postpartum Depression: Analysis from a Community Sample in Ghana
}

\author{
Abdul Cadri ${ }^{1,}$, Abdul Aziz Nagumsi Bonyo ${ }^{2}$, Aboagye Gyan Richard ${ }^{3}$, Augustine Adomah-Afari ${ }^{2}$ \\ ${ }^{1}$ Social and Behavioural Science, School of Public Health, University of Ghana, Legon- Accra, Ghana \\ ${ }^{2}$ Health Policy, Planning and Management, School of Public Health, University of Ghana, Legon- Accra, Ghana \\ ${ }^{3}$ Family and Community Health, School of Public Health, University of Health and Allied Sciences, Ho, Ghana
}

Email address:

abdul20c@yahoo.com (A. Cadri), meetaziz11@gmail.com (A. A. N. Bonyo), aboagyegyan94@gmail.com (A. G. Richard), augustineafari@yahoo.co.uk (A. Adomah-Afari), afari@ug.edu.gh (A. Adomah-Afari)

${ }^{*}$ Corresponding author

\section{To cite this article:}

Abdul Cadri, Abdul Aziz Nagumsi Bonyo, Aboagye Gyan Richard, Augustine Adomah-Afari. The Effect of Social Health Issues on Postpartum Depression: Analysis from a Community Sample in Ghana. Central African Journal of Public Health.

Vol. 6, No. 2, 2020, pp. 88-94. doi: 10.11648/j.cajph.20200602.16

Received: February 5, 2020; Accepted: February 21, 2020; Published: March 2, 2020

\begin{abstract}
Background: Postpartum depression is a mild mental or behavioural disorder that usually commences few weeks after delivery. Even though some studies have identified the association between postpartum depression and sociodemographic factors, no study has explored the association between stressors in women's life after birth and postpartum depression. Aims/objectives: This study aimed to assess the population prevalence of postpartum depression among women in the Hohoe Municipality, the prevalence of stressful life events and social health issues they experienced, and its association with postpartum depression. The coping strategies they adopted to enable them move on were also examined. Method: Analytical cross-sectional study design using quantitative techniques was employed in achieving the objectives of this study. A total of 172 participants who were between five and twelve weeks postpartum were recruited to respond to a structured questionnaire. The Edinburgh postnatal depression scale was used to measure depression. The 28 item Brief Cope Scale was used to examine the coping strategies postpartum depressed women adopted. Data were analysed using Stata version 14. Logistic regression model was used to test for association and statistical significance was tested at $\mathrm{p}<0.05$. Results: The prevalence of postpartum depression among the women in the study was $32.6 \%$. More than half of the respondents $(64.5 \%)$ reported experiencing stressful life events or social health issues after birth with $30.2 \%$ of them reporting one or two and $34.3 \%$ of them reporting to have experienced three or more stressful life events. Odds were raised in women who reported one or two $(\mathrm{AOR}=6.01,95 \% \mathrm{CI}=1.57-22.99)$ and three or more $(\mathrm{AOR}=32,95 \% \mathrm{CI}=8.33-124.06)$ stressful life events or social health issues than those who did not experience any social health issue; and the difference was statistically significant $(\mathrm{p}$ $<0.05$ ). Conclusion: The findings of the study indicated that the prevalence of postpartum depression was $32.6 \%$. Moreover, $64.5 \%$ of the women reported to have experienced some stressful life events. Experience of social health issues was found to be significantly associated with postpartum depression. There is the need for policy makers and healthcare practitioners to develop interventions that would comprehensively cater for the psychological health and wellbeing of postpartum mothers; and education on the right coping strategies.
\end{abstract}

Keywords: Depression, Social Health, Stressful Event, Coping

\section{Introduction}

Pregnancy and postpartum comes with intense physical and emotional changes, which may sometimes lead to mental disorders and symptoms ranging from mild to psychotic [1].
One out of every four women is expected to experience an episode of major depression in their lifetime, with the greatest incidence occurring during the reproductive years [2]. Research indicated that some of the risk factors linked to the development of postpartum depression (PPD) include prior history of depressive disorder and /or premenstrual 
dysphoric disorder, depression during pregnancy, inadequate social support and negative life events [2]. It is argued that mostly, mothers who suffer from postpartum depression often feel cheated and robbed of the first few months of their infants' lives and those who have experienced it describe feeling like "death warmed up" and being "afraid to be alive" [3].

Arguably, postpartum depression can result from a preexisting case of baby blues or can be apparent after the first few weeks of giving birth and can last as long as 14 months and the symptoms include anxiety, guilt, negative maternal attitudes, poor parenting, and self-efficacy [4]. Other studies observe that it may result from sleep disturbances, and in severe cases may have suicidal ideation [5]. Some studies have also shown that mothers who experience depressive episodes during pregnancy are at high risk of experiencing the depressive episodes again during the postpartum period, meaning there is an association between antenatal depression and postpartum depression [6].

Research has indicated that the prevalence of postpartum depression (PPD) ranges from $7.6 \%$ to $39 \%$ in various parts of the world [7]. It is suggested that the range of the prevalence internationally may be due to cross cultural variables, screening methods, different perception of mental health and its stigma, or difference in socio-economic backgrounds [4]. Even though the factors that predispose a woman to postpartum depression vary as well, usually, four main categories are identified; and they include socioeconomic, demographic, maternal and social support [4].

Other researchers posit that some stressful life events and social health issues that have an impact on maternal psychological morbidity include; death of a family member, serious illness or injury, housing problems, a lot of family argument or fight, being scared by other people's behaviour, having drug and alcohol problems, trouble with the police or having to go to the court, separation or divorce, homelessness, job loss, being abused and financial difficulty $[8,9]$. A small but gradually growing body of research emphasizes on postpartum mental health risk associated with intimate partner abuse [10]. For instance, a study found that women who experienced intimate partner abuse during pregnancy and after delivery (including physical, sexual, emotional, financial and neglect) reported rates of depression, anxiety and stress that were almost twice the rates reported by mothers who did not experience intimate partner abuse [10].

Different people adopt different coping strategies to enable them move on when experiencing stressful life events or social health issues [11]. Research has found that the coping strategies could either be active coping strategy or avoidant coping strategy [11]. These researchers explained that using active coping strategy is generally thought to be better and more adaptive way of dealing with stressful events while avoidance coping strategy is considered less adaptive. Active coping strategies include seeking social support, confrontive coping, positive reappraisal and planned problem solving whilst the avoidance coping strategies include distancing, self-control, accepting responsibility and escape or avoidance [12].

Another researcher also categorized coping mechanisms into Adaptive and Maladaptive coping strategies [13]. Adaptive coping strategies comprise active coping, instrumental support, planning, acceptance, emotional support, humour, positive reframing and religion. Maladaptive coping strategies on the other hand, include behavioural disengagement, denial, self-distraction, selfblame, substance abuse and venting. A growing body of research has indicated that many people adopt religious coping as their main strategy when stressed or depressed and found that people who use religious beliefs to cope with depression and stressful life events tend to handle their conditions more effectively than those who do not [14]. It was further argued that those who use religious coping strategies rely on a higher power and believe that what they are going through is meant to happen to them and their problem will be solved when the time is due and this in turn reduces the pressure to control circumstances and worry about results [14].

Notably, a study in rural Ghana established an association between antenatal depression and adverse consequences for the mother and new born [6]. However, it appears that limited evidence exists on the influence of stressful life events on postpartum depression among childbearing women in Ghana, especially in the Hohoe Municipality. Therefore, the purpose of this study was to examine the association between postpartum depression and stressful life events and social health issues experienced by women after birth as well as their corresponding coping strategies. This paper suggests the need for policy makers and healthcare practitioners to develop interventions that would comprehensively cater for the psychological health and wellbeing of postpartum mothers; and education on the right coping strategies.

\section{Materials and Methods}

\subsection{Study Setting}

The study was conducted in the Hohoe Municipality, which is one of the Municipal Assemblies of the Volta Region in Ghana. It has a population of 167,016 comprising of $52.1 \%$ females and $47.9 \%$ males. The total fertility rate is 3.3 in the municipality. According to the 2010 housing and population census, the municipality has a total land area of 1 , $172 \mathrm{~km}^{2}$ and located on longitude $0^{\circ} 15 \mathrm{E}$ and $0^{\circ} 45^{\prime} \mathrm{E}$ and latitude $6^{\circ} 45^{\prime} \mathrm{N}$ and $7^{\circ} 15^{\prime} \mathrm{N}$. Sixty-six percent of the population aged 15 years and above are economically active whilst the others are not [15].

\subsection{Study Design and Population}

Analytical cross-sectional study design using quantitative technique was employed for this study. The study included mothers aged 15-49 years who had delivered between five and twelve weeks and sought healthcare in the Hohoe Municipality. This is the period when most of them would be 
experiencing majority of the social health issues and stressful life events, if any. The study excluded women who had delivered less than five weeks or more than twelve weeks as well as women who had stillbirth or whose baby was known to have died. All the women who were available and signed an informed consent form were allowed to participate in the study.

\subsection{Sample Size Determination and Sampling Technique}

A prevalence of $11.3 \%$ postpartum depression was applied based on previous studies in Kintampo South and northern Ghana [16, 17]. Therefore, an assumption of $11.3 \%$ proportion was made of the study population. Using the formula for estimating a population proportion [18] and precision of $5 \%$, normal standard deviation of 1.96 at $95 \%$ confidence interval and a $10 \%$ non-response rate, a sample size of 172 was used for the study. Respondents who met the inclusion criteria were then randomly recruited for the study.

\subsection{Data Collection Procedure}

A structured questionnaire was designed to capture the socio-demographic characteristics of the postpartum mothers as well as the stressful life events, social health issues they had experienced after delivery, and coping strategies adopted using an interviewer-administered strategy. Standard tools such as the 10-item 'Edinburg Postpartum Depression Scale' and the 28-item Brief Cope Scale were used to assess the prevalence of depression among the mothers and the coping strategies they adopted respectively, and they were both Likert type scales. Another section captured data on stressful life events and social health issues and responses were either 'Yes' or 'No'. Trained research assistants administered the questions in selected facilities where the mothers went for Child Welfare Clinic.

\subsection{Data Analysis}

Data was entered using the EPIDATA version 3.1, UK software and exported to Stata version 14, US software for analysis. Data entered was scrutinized and cleaned and validated to ensure its quality. Data from the answered questionnaires was then coded and analysed based on the objectives of the study. Descriptive statistics as well as cross tabulations were used to analyse the data. In addition, Chi-square and Logistic regression were used to assess the association between the dependent and independent variables. The results were confined at 0.05 significance level. Moreover, tables and graphs were used to view the results comprehensively.

\section{Results}

\subsection{Demographic Characteristics of Respondents}

Out of the 172 respondents, $80(45.5 \%)$ were in the age range 25-34 years accounting for the highest proportion. In all, $166(96.5 \%)$ of the participants had a level of formal education ranging from primary to tertiary while only 6
(3.5\%) did not have any form of formal education. Majority, $166(96.5 \%)$ of the respondents were Christians while 6 (3.5\%) were Muslims. Similarly, $122(70.9 \%)$ were married, $43(25.0 \%)$ were single and $7(4.1 \%)$ were cohabiting. Majority of the respondents, $76(44.2 \%)$ were into trading while a few of them $16(9.3 \%)$ were civil servants. The vast majority, $148(86 \%)$ of the respondents were Ewes (Table 1).

Table 1. Demographic characteristics of respondents.

\begin{tabular}{|c|c|c|}
\hline Characteristic & Frequency & Percentage (\%) \\
\hline Number recruited & 172 & 100 \\
\hline \multicolumn{3}{|l|}{ Age (years) } \\
\hline $15-24$ & 61 & 35.5 \\
\hline $25-34$ & 80 & 45.5 \\
\hline $35-49$ & 31 & 18.0 \\
\hline \multicolumn{3}{|l|}{ Education } \\
\hline None & 6 & 3.5 \\
\hline Primary & 16 & 9.3 \\
\hline JHS/Middle School & 102 & 59.3 \\
\hline SHS/ Secondary School & 26 & 15.1 \\
\hline Tertiary & 22 & 12.8 \\
\hline \multicolumn{3}{|l|}{ Marital status } \\
\hline Single & 43 & 25.0 \\
\hline Married & 122 & 70.9 \\
\hline Co-habitation & 7 & 4.1 \\
\hline \multicolumn{3}{|l|}{ Occupation } \\
\hline Unemployed & 34 & 19.8 \\
\hline Trading & 76 & 44.2 \\
\hline Artisan & 46 & 26.7 \\
\hline Civil servant & 16 & 9.3 \\
\hline \multicolumn{3}{|l|}{ Religion } \\
\hline Christianity & 166 & 96.5 \\
\hline Islam & 6 & 3.5 \\
\hline \multicolumn{3}{|l|}{ Ethnicity } \\
\hline Ewe & 148 & 86.0 \\
\hline Akan & 10 & 5.8 \\
\hline Hausa & 8 & 4.7 \\
\hline Others & 6 & 3.5 \\
\hline
\end{tabular}

\subsection{Prevalence of Postpartum Depression}

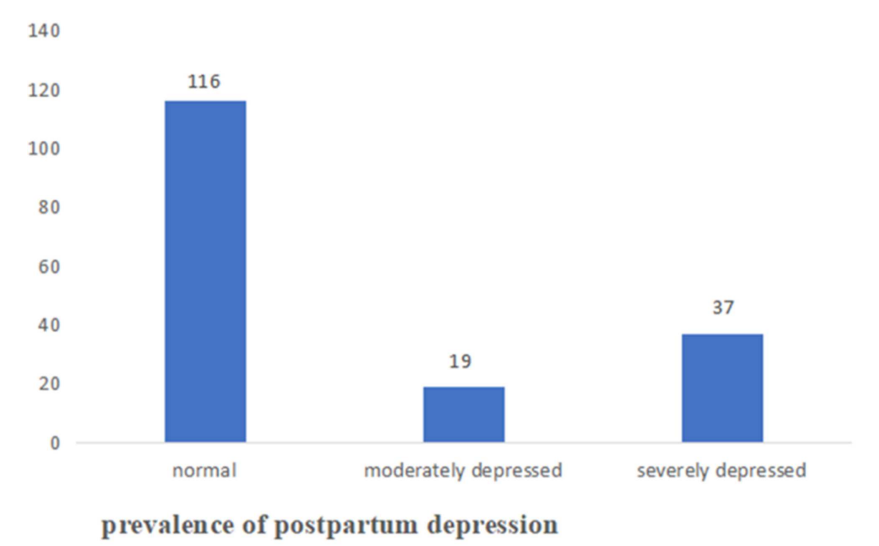

Figure 1. Prevalence of postpartum depression.

Figure 1 presents the results of the prevalence of postpartum depression among the respondents. Out of the 172 , more than half of them $(67.4 \%)$ had a total depression 
score that was less than 10 . A depression score between 10 and 12 was reported in $11 \%$ of them and $21.6 \%$ of them had a depression score that was 13 or more. In general, 116 $(67.4 \%)$ of them were normal while $56(32.6 \%)$ of them reported a form of depression ranging from moderate to severe.

\subsection{Prevalence of Stressful Life Events and Social Health Issues}

Figure 2 presents results of the prevalence of social health issues and stressful life events among the respondents. The key themes measured included financial stressors, emotional stressors, traumatic stressors and relationship stressors. While $61(35.5 \%)$ of the respondents had not experienced any form of stressful life events or social health issues after birth, 52 $(30.2 \%)$ of them had experienced one or two stressful life events or social health issues after birth, and 59 (34.3\%) had experienced three or more stressful life events or social health issues after birth.

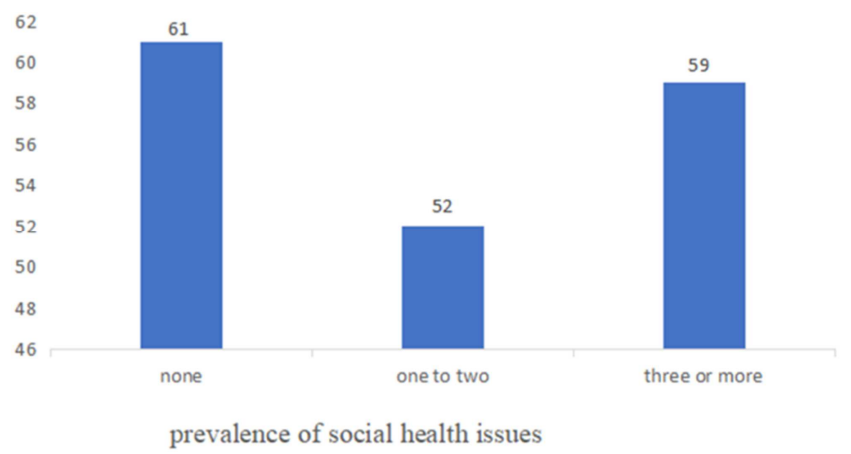

Figure 2. Prevalence of stressful life events and social health issues.

\subsection{Effect of Social Health Issues on Postpartum Depression}

Table 2 shows the results of the association between postpartum depression and social health issues experienced after birth. The results showed that 58 (95.1\%) of those who had not experienced any social health issue reported a normal level of postpartum depression while $3(4.9 \%)$ of them reported a form of postpartum depression. With regards to those who reported one to two social health issues, 37 $(71.2 \%)$ were normal whilst $15(28.8 \%)$ of them reported a form of postpartum depression. In respondents who reported three or more social health issues, 21 (35.6\%) were normal while $38(64.4 \%)$ of them reported a form of postpartum depression. The difference between the number of social health issues experienced and postpartum depression was statistically significant $\left(\chi^{2}=48.8049 ; \mathrm{p}=<0.001\right)$.

\subsection{Logistic Regression Model of Factors Associated with the Risk of Postpartum Depression}

The results showed that age, occupation, marital status and social health issues were significantly associated with postpartum depression in the unadjusted model $(\mathrm{p}<0.05)$. However, after adjusting for the effect of other variables, occupation and marital status were not significantly associated with postpartum depression $(p>0.05)$. The age range 25-34 years was also not significantly associated with postpartum depression in the adjusted model.

Experience of social health issues was found to be significantly associated with postpartum depression in both the adjusted and unadjusted logistic regression analysis. Respondents who experienced one or two social health issues had an odds ratio of $7.84(95 \% \mathrm{CI} ; 2.12-28.94$; $<<0.001)$ in the unadjusted logistic regression analysis and they had an odds ratio of $6.01(95 \% \mathrm{CI} ; 1.57-22.99 ; \mathrm{p}=0.009)$ in the adjusted logistic regression analysis whilst those who experienced three or more had an odds ratio of $34.98(95 \%$ CI; 9.75-125.45; $\mathrm{p}<0.001)$ in the unadjusted logistic regression analysis and they had an odds ratio of 32 (95\% CI; $8.33-124.06 ; \mathrm{p}<0.001)$ in the logistic regression analysis when compared with the reference group (Table 3 ).

\subsection{Coping Strategies Adopted by Postpartum Depressed Women}

Table 4 presents results of the coping strategies adopted by postpartum depressed women and those who had experienced social health issues and stressful life events after birth to enable them move on. The respondents did not use a single coping strategy but tend to use two or more of the coping strategies.

In most cases, the coping strategy used was religion [65 $(89.0 \%)]$. Active coping was used by $41(56.2 \%)$ of the respondents. Furthermore, $32(42.5 \%)$ of the respondents tend to use self-distraction coping strategy, and 44 (60.3\%) used emotional support. Additionally, planning, as a coping strategy was used by $40(54.8 \%)$ of the respondents, and 20 (27.4\%) also used denial as a coping strategy. The coping strategy that was least used by the respondents was substance use, which recorded only 7 (9.6\%).

Table 2. Effect of social health issues on postpartum depressed.

\begin{tabular}{|c|c|c|c|c|c|}
\hline \multirow{2}{*}{ Characteristic } & \multicolumn{5}{|c|}{ Social health issues experienced } \\
\hline & None N (\%) & One to two N (\%) & Three or more N (\%) & $\chi^{2}$ & p-value \\
\hline Normal & $58(95.1)$ & $37(71.2)$ & $21(35.6)$ & & \\
\hline Depressed & $3(4.9)$ & $15(28.8)$ & $38(64.4)$ & 48.8049 & $<0.001$ \\
\hline
\end{tabular}


Table 3. Logistic regression model of factors associated with the risk of postpartum depression.

\begin{tabular}{|c|c|c|c|c|c|}
\hline \multirow{2}{*}{ Variable } & \multirow{2}{*}{ Depressed N (\%) } & \multicolumn{2}{|c|}{ Unadjusted logistic regression } & \multicolumn{2}{|c|}{ Adjusted logistic regression } \\
\hline & & Odds ratio $(95 \% \mathrm{CI})$ & p-value & Odds ratio $(95 \% \mathrm{CI})$ & p-value \\
\hline \multicolumn{6}{|l|}{ Age group } \\
\hline $15-24$ & $29(51.8)$ & $1.00 \mathrm{ref}$ & & & \\
\hline $25-34$ & $23(41.1)$ & $0.44(0.22-0.89)$ & & $0.45(0.18-1.16)$ & 0.099 \\
\hline $35-49$ & $4(7.1)$ & $0.16(0.05-0.52)$ & 0.002 & $0.12(0.03-0.51)$ & 0.004 \\
\hline \multicolumn{6}{|l|}{ Occupation } \\
\hline Unemployed & $13(23.2)$ & $1.00 \mathrm{ref}$ & & & \\
\hline Trading & $30(53.6)$ & $1.05(0.46-2.41)$ & & $2.57(0.81-8.21)$ & 0.110 \\
\hline Artisan & $12(21.4)$ & $0.57(0.22-1.48)$ & & $2.14(0.56-8.16)$ & 0.265 \\
\hline Civil servant & $1(1.8)$ & $0.11(0.01-0.91)$ & 0.022 & $0.68(0.06-7.31)$ & 0.750 \\
\hline \multicolumn{6}{|l|}{ Marital status } \\
\hline Single & $23(41.1)$ & $1.00 \mathrm{ref}$ & & & \\
\hline Married & $31(55.4)$ & $0.30(0.14-0.61)$ & & $0.60(0.21-1.63)$ & 0.309 \\
\hline Co-habiting & $2(3.6)$ & $0.34(0.06-1.99)$ & 0.004 & $0.61(0.06-5.33)$ & 0.654 \\
\hline \multicolumn{6}{|l|}{ Religion } \\
\hline Christianity & $53(94.6)$ & & & & \\
\hline Islam & $3(5.4)$ & $2.13(0.42-10.92)$ & 0.368 & & \\
\hline \multicolumn{6}{|c|}{ Social health issues } \\
\hline None & $3(5.4)$ & $1.00 \mathrm{ref}$ & & & \\
\hline $1-2$ & $15(26.8)$ & $7.84(2.12-28.94)$ & & $6.01(1.57-22.99)$ & 0.009 \\
\hline $3+$ & $38(67.9)$ & $34.98(9.75-125.45)$ & $<0.001$ & $32(8.33-124.06)$ & $<0.001$ \\
\hline
\end{tabular}

Table 4. Coping strategies adopted by postpartum depressed women.

\begin{tabular}{lll}
\hline Characteristic & Frequency & Percentage (\%) \\
\hline Self-distraction & 32 & 42.5 \\
Active coping & 41 & 56.2 \\
Denial & 20 & 27.4 \\
Substance abuse & 7 & 9.6 \\
Emotional support & 44 & 60.3 \\
Instrumental support & 47 & 64.4 \\
Behavioural & 12 & 16.4 \\
disengagement & 24 & 32.9 \\
Venting & 16 & 21.9 \\
Positive reframing & 40 & 54.8 \\
Planning & 30 & 41.1 \\
Humour & 23 & 31.5 \\
Acceptance & 65 & 89.0 \\
Religion & 31 & 42.5 \\
Self-blame & & \\
\hline
\end{tabular}

\section{Discussion}

This study found that at five to twelve weeks postpartum, almost one-third $(32.6 \%)$ of the respondents reported a case of PPD. The prevalence of PPD found in this study was quite similar to a study that was conducted in Zimbabwe [19]. The Edinburgh Postnatal Depression scale was used in that study and at six to ten weeks postpartum, it was found that about $30.5 \%$ of the respondents had experienced a form of postpartum depression. A study conducted in the Kumasi Metropolis in Ghana found that $22.3 \%$ of the respondents had experienced a form of PPD at six weeks, which was lower than the prevalence found in this current study [1]. The difference in the prevalence could be due to the difference in study settings.

This study found that age was significantly associated with postpartum depression $(p<0.05)$. This finding confirms the findings of a study, which reported that maternal age was greatly associated with risk of antepartum and postpartum depression [20]. In this study, the comparison in the various age groups showed that the level of depression was high in the age group 14-24 years. This could possibly be because a substantial proportion of the respondents in that age group might have given birth as a result of unwanted pregnancies. In addition, some of the respondents might be single mothers without neither a partner nor income and might be going through a lot of stress from family members. Furthermore, the study found that marital status of the women was also associated with PPD. This finding is in conformity with a study, which also reported that marital status was a significant predictor of postpartum depression [21]. Whereas being a single mother can have a negative effect on mothers, being in a bad relationship can have a great deal of effect as well.

Findings of this study showed that majority of the respondents $(64.5 \%)$ reported to have experienced a stressful life event or a social health issue, with $30.2 \%$ of them reporting to have experienced one or two and $34.3 \%$ of them reporting to have experienced three or more. Experience of stressful life events during the postpartum period is similar to earlier findings in Australian population [8]. However, in that study, majority of the mothers $(52.3 \%)$ did not experience any stressful life event or social health issues after birth as compared to this study in which majority (64.5\%) reported to have experienced a stressful life event or a social health issue after birth. The difference in findings could be due to differences in the socio-cultural variables.

The findings of this study further showed that experiencing one or two stressful life events or social health issues during the postpartum period was significantly associated with about six-fold increase in odds of depressive symptoms whilst experiencing three or more stressful life events or social health issues during the postpartum period was significantly associated with about thirty two-fold increase in odds of depressive symptoms. The findings of this study were in 
accordance with a study, which reported that experiencing high levels of stressful life events was related to high levels of depressive symptoms [22].

The findings of this study draw attention to stressful life events and social health issues in women's life at the postpartum period that may put them at risk of poor mental health outcomes. It was found that majority of the women in the postpartum depressed group had experienced some social health issues.

Additionally, the findings of this study indicated that the coping strategy that was mostly used among the women who were stressed or depressed was religion while substance use was the coping strategy that was least used among them. Instrumental support, emotional support, active coping, and planning were the coping strategies used mostly after religion in descending order. These coping strategies that were frequently used among the depressed or stressed group falls into adaptive coping strategy as categorised in a previous study [13]. Maladaptive coping strategies like substance abuse, behavioural disengagement, and denial were also used but not frequently in this study. It is important that the mothers are encouraged to stick to adaptive coping strategies rather than the maladaptive strategies when they are stressed or depressed.

\section{Conclusions}

The study examined the association between postpartum depression and stressful life events as well as social health issues experienced and the appropriate coping strategies adopted by women after birth. The findings of the study indicated that the prevalence of postpartum depression was $32.6 \%$. Moreover, $64.5 \%$ of the women reported to have experienced some stressful life events with $30.2 \%$ of them reporting one or two stressful life event or social health issues, and $34.3 \%$ reported three or more stressful life events or social health issues after birth.

Experience of social health issues was found to be significantly associated with postpartum depression. Respondents adopted a range of coping strategies and the most widely used coping strategy was religion while the least used was substance use. The findings of the study suggest the need for policy makers and healthcare practitioners to develop interventions that would comprehensively cater for the psychological health and wellbeing of postpartum mothers; and education on the right coping strategies recommended.

\section{Limitations}

The cross-sectional nature of the study was not appropriate to establish a causal relationship between dependent variable and independent variables. There was a potential recall bias by the respondents in providing responses to questions. Future research should seek to increase the sample size as well as include other districts, municipalities, and metropolitan Assemblies in order to increase the potential for generalisation of the results.

\section{References}

[1] Buabeng, A. A. (2015). Risk factors influencing postpartum depression among women attending postnatal clinic at Komfo Anokye Teaching Hospital, Kumasi, Ghana. Thesis, Kwame Nkrumah University of Science and Technology, Kumasi, Ghana.

[2] Hatton, D. C., Harrison-Hohner, J., Coste, S., Dorato, V., Curet, L. B., \& McCarron, D. A. (2005). Symptoms of postpartum depression and breastfeeding. Journal of Human Lactation, 21 (4), 444-449.

[3] Beck, C. T. (2002). Postpartum depression: A metasynthesis. Qualitative Health Research, 12 (4), 453-472.

[4] Lanes, A., Kuk, J. L., \& Tamim, H. (2011). Prevalence and characteristics of postpartum depression symptomatology among Canadian women: a cross-sectional study. BMC Public Health, 11 (1), 302.

[5] Al Hinai, F. I., \& Al Hinai, S. S. (2014). Prospective study on prevalence and risk factors of postpartum depression in Aldakhliya governorate in oman. Oman Medical Journal, 29 (3), 198.

[6] Weobong, B., ten Asbroek, A. H., Soremekun, S., Manu, A. A., Owusu-Agyei, S., Prince, M., \& Kirkwood, B. R. (2014). Association of antenatal depression with adverse consequences for the Mother and Newborn in Rural Ghana: findings from the DON population-based cohort study. PloS one, 9 (12), e116333.

[7] Poomalar, G. K., \& Arounassalame, B. (2014). Impact of socio-cultural factors on postpartum depression in South Indian women. International Journal of Reproduction, Contraception, Obstetrics and Gynecology, 3 (2), 338-343.

[8] Yelland, J., Sutherland, G., \& Brown, S. J. (2010). Postpartum anxiety, depression and social health: findings from a population-based survey of Australian women. BMC Public Health, 10 (1), 771.

[9] Weetra, D., Glover, K., Buckskin, M., Kit, J. A., Leane, C., Mitchell, A.,... \& Brown, S. J. (2016). Stressful events, social health issues and psychological distress in Aboriginal women having a baby in South Australia: implications for antenatal care. BMC Pregnancy and Childbirth, 16 (1), 88.

[10] Desmarais, S. L., Pritchard, A., Lowder, E. M., \& Janssen, P. A. (2014). Intimate partner abuse before and during pregnancy as risk factors for postpartum mental health problems. BMC Pregnancy and Childbirth, 14 (1), 132.

[11] Glasscock, D. J., Andersen, J. H., Labriola, M., Rasmussen, K., \& Hansen, C. D. (2013). Can negative life events and coping style help explain socioeconomic differences in perceived stress among adolescents? A cross-sectional study based on the West Jutland cohort study. BMC Public Health, $13(1), 532$.

[12] Bardwell, W. A., Ancoli-Israel, S., \& Dimsdale, J. E. (2001). Types of coping strategies are associated with increased depressive symptoms in patients with obstructive sleep apnea. Journal of Sleep and Sleep Disorders Research, 24 (8), 905909. 
[13] Kasi, P. M., Naqvi, H. A., Afghan, A. K., Khawar, T., Khan, F. H., Khan, U. Z. \& Khan, H. M. (2012). Coping styles in patients with anxiety and depression. ISRN psychiatry, 2012.

[14] Aflakseir, A., \& Mahdiyar, M. (2016). The role of religious coping strategies in predicting depression among a sample of women with fertility problems in Shiraz. Journal of Reproduction \& Infertility, 17 (2), 117.

[15] GSS Ghana Statistical Service. (2013). 2010 Population \& Housing Census Report Women \& Men in Ghana.

[16] Weobong, B., ten Asbroek, A. H., Soremekun, S., Gram, L., Amenga-Etego, S., Danso, S.. \& Kirkwood, B. R. (2015). Association between probable postnatal depression and increased infant mortality and morbidity: findings from the DON population-based cohort study in rural Ghana. BMJ Open, 5 (8), e006509.

[17] Scorza, p., Owusu-Agyei, S., asampong, E., \& Wainberg, M. L. (2015). The expression of perinatal depression in rural Ghana. International Journal of Culture and Mental Health, 8 (4), 370-381.
[18] Snedecor, G. W., Cochran, W. G (1989). Statistical methods, 8th Ed: Lowa State Press.

[19] January, J., Mutamba, N., \& Maradzika, J. (2017). Correlates of postnatal depression among women Zimbabwean semiurban and rural settings. Journal of Psychology in Africa, 27 (1), 93-96.

[20] Rich-Edwards, J. W., Kleinman, K., Abrams, A., Harlow, B. L., McLaughlin, T. J., Joffe, H., \& Gillman, M. W. (2006). Sociodemographic predictors of antenatal and postpartum depressive symptoms among women in a medical group practice. Journal of Epidemiology and Community Health, 60 (3), 221-227.

[21] Segre, L. S., O’Hara, M. W., Arndt, S., \& Stuart, S. (2007). The prevalence of postpartum depression. Social Psychiatry and Psychiatric Epidemiology, 42 (4), 316-321.

[22] Sokratous, S., Merkouris, A., Middleton, N., \& Karanikola, M. (2013). The association between stressful life events and depressive symptoms among Cypriot university students: a cross-sectional descriptive correlational study. BMC Public Health, 13 (1), 1121. 